\title{
In Situ Ti Isotopic Measurements by Laser Ablation MC-ICP-MS
}

\author{
Hsin-Wei Chen ${ }^{1,{ }^{*}}$, Typhoon Lee ${ }^{2}$, Der-Chuen Lee ${ }^{2}$, and Yoshiyuki Iizuka ${ }^{2}$ \\ ${ }^{1}$ Institute of Geosciences, National Taiwan University, Taipei, Taiwan, ROC \\ ${ }^{2}$ Instutute of Earth Sciences, Academia Sinica, Taipei, Taiwan, ROC
}

Received 10 July 2008, accepted 19 September 2008

\begin{abstract}
We have been developing Laser Ablation Multi-Collector Inductively Coupled Plasma Mass Spectrometry (MC-ICP-MS) technique to measure titanium isotopic composition in situ. A principal aim of this work is to search for isotopic heterogeneities larger than the few epsilons $\left(\varepsilon\right.$, in parts in $\left.10^{4}\right)$ of the solar system. Our analytical precision of the ratios of ${ }^{46} \mathrm{Ti}$, ${ }^{48} \mathrm{Ti}$, and ${ }^{50} \mathrm{Ti}$ to ${ }^{49} \mathrm{Ti}$ after exponential-law mass discrimination correction normalizing ${ }^{47} \mathrm{Ti} /{ }^{49} \mathrm{Ti}$ to 1.33375 were about $2.5 \varepsilon(2 \sigma)$. Mixture solutions were prepared by adding the expected level of $\mathrm{Ca}, \mathrm{Cr}, \mathrm{Mg}$, and $\mathrm{Al}$ to the Ti solutions to demonstrate that our interference correction is effective. We then applied our technique with $213 \mathrm{~nm} \mathrm{Nd}$-YAG laser ablation to five Ti-rich terrestrial solids, and all of them also showed titanium isotopic composition that was consistent with one another and agreed with that for the solution standard. It appears that the in situ laser technique did not significantly increase the long-term reproducibility beyond the $2.5 \varepsilon$ established using the solution method. This is an order of magnitude better than the typical precision of a few permil for secondary ion mass spectrometry (SIMS). The combination of the ability to perform in situ analysis on $30 \mu \mathrm{m}$ spots with $\varepsilon$ level precision is a niche for LA-MC-ICP-MS. We also ablated two lines on a fassaite grain from a large well studied CAI Egg-6 of the Allende meteorite. After the mass discrimination was corrected by normalizing ${ }^{47} \mathrm{Ti} /{ }^{49} \mathrm{Ti}$, the ${ }^{46} \mathrm{Ti}$ and ${ }^{48} \mathrm{Ti}$ are normal within about $2 \varepsilon$ while ${ }^{50} \mathrm{Ti} /{ }^{49} \mathrm{Ti}$ shows a $9 \varepsilon$ excess. These data are in excellent agreement with thermal ionization mass spectrometry (TIMS) results. Comparing our ICP-MS results against the results from TIMS studies, we found that our normal titanium isotopic ratios were closest to the less precise data of Heydegger et al. (1979) who measured $\mathrm{Ti}^{+}$. We support the proposal to IUPAC to change the accepted Ti abundance to that measured by ICP-MS and TIMS without using Ti oxides.
\end{abstract}

Key words: Titanium isotopes, Laser Ablation, MC-ICP-MS, CAI

Citation: Chen, H. W., T. Lee, D. C. Lee, and Y. Iizuka, 2009: In situ Ti isotopic measurements by Laser Ablation MC-ICP-MS. Terr. Atmos. Ocean. Sci., 20, 703-712, doi: 10.3319/TAO.2008.09.19.01(TT)

\section{INTRODUCTION}

During the past several decades, titanium isotopic compositions of meteorites have been studied to constrain their nucleosynthetic origins and mixing in the early solar system. The five titanium stable isotopes are thought to have come from several distinct stellar processes; and the refractory material makes Ti one of the earliest elements to condense in the solar system, or perhaps the last residue of pre-solar material to evaporate. Pioneering studies started from the measurements of isotopic composition of bulk chondrites, $\mathrm{Ca}-\mathrm{Al}$ rich inclusions (CAIs), and chondrules by conventional thermal ionization mass spectrometry (TIMS) [Heydegger et al. 1979 (hereafter, HFC); Niederer et al.

\footnotetext{
* Corresponding author

E-mail:haart@earth.sinica.edu.tw
}

1980, 1981, 1985 (hereafter, NPW); Niemeyer and Lugmair 1981, 1984 (hereafter, NL); Niemeyer 1988 (hereafter, N); Papanastassiou and Brigham 1989], and found the endemic positive ${ }^{50} \mathrm{Ti}$ anomalies (less than 1.5 permil) in normal refractory inclusions and variable anomalies in all Ti isotopes in rare FUN inclusions ranging from -20.3 to 3.8 permil for ${ }^{50} \mathrm{Ti}$. Another approach focused on the in situ analysis of individual tiny titanium-bearing phases, such as hibonites by secondary ionization mass spectrometric techniques (SIMS) (Hutcheon et al. 1983; Fahey et al. 1985; Ireland and Compston 1985; Hinton et al. 1987). However, limited by analytical precision, they could only distinguish the larger variation in ${ }^{50} \mathrm{Ti}$ mostly in what was called "Platy hibonites" phases $\left({ }^{50} \mathrm{Ti}\right.$ anomalies from -68 to 273 permil). Therefore, Ti anomalies seem to be another vivid example 
of the cosmochemists' rule of thumb: "The smaller the sample size, the larger the isotopic heterogeneity" (for an incompletely mixed solar system, Lee 1988)!

Heydegger et al. (1982) were the first to notice the inconsistency among the titanium isotopic ratios of terrestrial standards published by different groups. They proposed that inter-laboratory inconsistency might be due to isobaric interference corrections (as large as $2.9 \%$ for ${ }^{50} \mathrm{Ti} /{ }^{49} \mathrm{Ti}$ due to interferences of ${ }^{48} \mathrm{Ti}^{18} \mathrm{O}^{+}$and ${ }^{49} \mathrm{Ti}^{17} \mathrm{O}^{+}$on ${ }^{50} \mathrm{Ti}^{16} \mathrm{O}^{+}$) using different chemical procedures and mass spectrometric techniques. NPW and NL measured $\mathrm{Ti}$ as $\mathrm{TiO}^{+}$instead of $\mathrm{Ti}^{+}$, which HFC used, to get enhanced signals (typically a factor of 20) and the precision can reach as high as epsilon $(\varepsilon, 1$ part in $10^{4}$ ) level. NL reported their titanium isotopic ratios as the deviation of ${ }^{\mathrm{i}} \mathrm{TiO} /{ }^{46} \mathrm{TiO}$ of samples relative to normal standards, apparently ignoring interference corrections, so their work had to carefully maintain the same oxygen isotopic composition for each individual run. The NPW presented data were corrected for isobaric $\mathrm{TiO}^{+}$interferences using oxygen isotopic composition as determined by Nier (1950).

Recently developed multi-collector inductively coupled plasma mass spectrometry (MC-ICP-MS) measurements provide excellent ionization efficiency, high precision isotopic data for many elements. The dominant mass discrimination process in ICP-MS is believed to take place when the sample ions try to squeeze through the central orifices of the sampling and skimming cones competing against $\mathrm{Ar}^{+}$into the flight tube (Freedman 2002). Unlike evaporation and ionization in TIMS, this process occurring in ICP-MS is not time dependent. Moreover, because for many elements the ionization efficiency is close to $100 \%$, thus the fractionations are almost only dependent on mass alone. For instance, neighboring elements such as $\mathrm{Ti}$ and $\mathrm{Cr}$ would undergo similar fractionation. This time and chemistry independent behavior for ICP-MS opens a new window to distinguish natural isotopic fractionation from instrumental mass discrimination effects measured with respect to a reference standard (e.g., Halliday et al. 1998). So far titanium isotopic measurements by ICP-MS have been limited to chemically extracted and purified sample solutions of several micro-grams in size initially (Zhu et al. 2002) but later reduced to hundreds of nanogram (Leya et al. 2007). The reported precision and accuracy were very high, typically less than $1 \varepsilon(2 \sigma)$. However, in order to achieve higher quality data, it becomes more important to remove any interferences. Higher mass resolution would be useful as well. For example, Leya et al. (2007) used a custom-made large-sized MC-ICP-MS (Nu 1700) that can partially resolve interferences such as $\mathrm{ArN}$, thus reducing the interference.

Combined with laser ablation techniques, this new tool can explore both the mass-dependent (linear) and massindependent (non-linear) isotopic effects of natural materi- als with higher precision ( $\varepsilon$ level) than those on previous SIMS techniques without chemical pre-treatments. Compared with previous TIMS studies, it was possible to understand this discrepancy of titanium isotopic ratios by using the constant instrument mass discrimination characteristic of ICP-MS. In principle, the mass discrimination behavior would remain roughly constant while the instrument condition was setup, irrespectively of analyzed elements (Halliday et al. 1998). It might act as a bridge to determine the instrumental discrimination factor by neighboring chromium, the element whose isotopic ratios are consistent among different groups, to examine the accuracy of those titanium reference ratio used on TIMSs for fractionation normalization. Hence, we expect the instrumental mass discrimination effect derived from titanium to be very close to the value derived from chromium measurements.

For the in situ investigation, laser ablation can utilize samples faster than SIMS, and enhance ion beam currents. E. D. Young's group at the University of California, Los Angeles (UCLA) has successfully performed in situ isotopic analysis of magnesium and silicon in meteoritic samples (Young et al. 2002; Simon et al. 2004; Shahar and Young 2007), and this success demonstrates the potential of LA-MC-ICP-MS. In this paper, we report on our ongoing effort on establishing the methodology using LAMC-ICP-MS. We show that our current set up is capable of measuring in situ titanium isotopic composition to the precision of $2-3 \varepsilon$ that SIMS is not currently able to achieve.

\section{EXPERIMENTAL METHODS}

\subsection{Sample Preparation}

A Ti standard solution with a concentration of $100 \mathrm{ppb}$ was prepared from high purity Merck CertiPUR grade Ti reagent. It was diluted to a $25 \mathrm{ppb}$ Ti solution standard that gave a Ti ion beam with intensity close to what was typical for LA-MC-ICP-MS isotopic analysis. A series of mixed solutions were also prepared to cover the range of interferences on Ti measurements from $\mathrm{Cr}, \mathrm{Ca}$, and $\mathrm{Al}-\mathrm{Mg}$ to test the performance of our interference correction procedure and possible matrix effects. For LA-MC-ICP-MS analysis, we prepared five titanium-bearing terrestrial solid phases: metal, rutile $\left(\mathrm{TiO}_{2}\right)$, sphene $(\mathrm{CaTiSiO})$, perovskite $\left(\mathrm{CaTiO}_{3}\right)$, and man-made fassaite glass to set up the LA-MC-ICP-MS analytical procedures. We also prepared a fassaite grain from Allende CAI Egg-6, whose bulk Ti isotopic composition was determined previously by TIMS, in order to check our results against the traditional technique.

\subsection{Mass Spectrometry}

The titanium solution standards were fed through a CETAC Aridus microconcentric desolvation nebulizer (Type 
T1H) under a constant 60 psi Ar pressure that gave an average sample uptake rate of about $0.08 \mathrm{ml} \mathrm{min}^{-1}$. The Ar sweep gas flow varied from 3.9 to $4.51 \mathrm{~min}^{-1}$ to optimize signal intensity and stability. To avoid forming huge $\mathrm{ArN}^{+}$interference, no additional $\mathrm{N}_{2}$ was supplied. Sample aerosols were then introduced into the plasma torch (Type Nu102O) with a coolant and auxiliary flow rates of 13 and $0.81 \mathrm{~min}^{-1}$, respectively. Ti was ionized by energy transfer from excited Ar atoms which were heated by the oscillating field energized by the $1300 \mathrm{~W}$ RF generator. Ti ions would then fly through the sampler cone (Type 319-541B) and the skimmer cone (Type 319-540) to form a stable $\mathrm{Ti}^{+}$signal of 35 45 pico-ampere, equivalent to about $0.7-0.9 \%$ of ion yield efficiency (signal detected/sample introduced), significantly higher than the best value of $0.1 \%$ offered by TIMS techniques (Niederer et al. 1981). A typical run for solution standards consumed about $5 \mathrm{ng}$ of Ti.

The center piece of our in situ analysis setup is a New Wave UP-213 laser ablation system which is based on a Nd-YAG solid state laser operating at $213 \mathrm{~nm}$, up-converted to five times of its fundamental wavelength of 1.064 micron. Argon was used as the carrier gas $\left(1.10 \sim 1.251 \mathrm{~min}^{-1}\right)$ and the ablated sample was directly introduced to the torch of our $\mathrm{Nu}$ Plasma MC-ICP-MS. The laser beam was operated at the "image aperture mode" to maintain a constant energy flux of $12-15$ joule $\mathrm{cm}^{-2}$ on the sample surface, gave laser pulses at repetition rates of $5-10 \mathrm{~Hz}$ to etch approximately $0.03 \mu \mathrm{m}$ in depth per shot on the ablated surface. The laser beam size and its scan speed were adjusted depending on the titanium concentration and the area of target to ensure roughly the same intensities of titanium signals for all analysis. For a typical $4 \%$ $\mathrm{TiO}_{2}$ content of meteoritic fassaite, $30 \mu \mathrm{m}$ of laser beam with $10 \mathrm{~Hz}$ of repetition rate can provide the same Ti consumption rate as a $25 \mathrm{ppb}$ Ti standard solution. We are aware of the benefit of using helium as the carrier gas in many cases, perhaps because it could rapidly remove thermal energy from the laser induced plasma to inhibit the condensation of small particles (Horn and Günther 2003) and reduce ablated particles stalled back on the sample surface (Mank and Mason 1999). However, it also caused an order of magnitude high background at 44, 50, and $54 \mathrm{amu}$, making it difficult to determine the Ti isotopic ratios precisely because we could no longer reliably monitor ${ }^{44} \mathrm{Ca}$ to correct the interferences of ${ }^{46} \mathrm{Ca}$ and ${ }^{48} \mathrm{Ca}$ on ${ }^{46} \mathrm{Ti}$ and ${ }^{48} \mathrm{Ti}$.

We used three magnetic jumping steps to cover the mass spectrum between 44 and $54 \mathrm{amu}$. The first step (46$50 \mathrm{amu}$ ) covers the five Ti peaks. The second step (50 $54 \mathrm{amu})$ covers the monitoring peaks $\left({ }^{53} \mathrm{Cr},{ }^{51} \mathrm{~V}\right)$ for the interferences $\left({ }^{50} \mathrm{Cr},{ }^{50} \mathrm{~V}\right)$ on ${ }^{50} \mathrm{Ti}$, and the third step (44 - $\left.48 \mathrm{amu}\right)$ covers the monitoring peaks ${ }^{44} \mathrm{Ca}$ for the interferences ${ }^{46} \mathrm{Ca}$ and ${ }^{48} \mathrm{Ca}$. Both the $50 \mathrm{amu}$ signal of the second step and $48 \mathrm{amu}$ signal of the last step were used to calibrate their intensities equivalent to the first step. Because of potential influence from elemental $\left(\mathrm{Ca}^{+}, \mathrm{Cr}^{+}\right)$and molecular $\left(\mathrm{ArC}^{+}\right.$,
$\mathrm{ArN}^{+}$) interferences on 46,48 , and $50 \mathrm{amu}$, we normalized the ${ }^{47} \mathrm{Ti} /{ }^{49} \mathrm{Ti}$ to the TIMS value of 1.33375 (Niederer et al. 1981) to correct for instrumental mass discrimination using the exponential mass fractionation law (Russell et al. 1978).

For normal Ti solution analysis, the instrumental zeros were measured by ESA deflection (baselines signal), and the molecular interference of ${ }^{36} \mathrm{Ar}^{14} \mathrm{~N}^{+}$and ${ }^{36} \mathrm{Ar}^{12} \mathrm{C}^{+}$on ${ }^{50} \mathrm{Ti}$ and ${ }^{48} \mathrm{Ti}$ were corrected by monitoring ${ }^{40} \mathrm{Ar}^{14} \mathrm{~N}$ and ${ }^{40} \mathrm{Ar}^{12} \mathrm{C}$, whose signals were generally less than $5 \times 10^{-13}$ and $2 \times$ $10^{-13}$ Amp, respectively. The instrumental zeros of several reagent mixtures and laser ablation measurements were determined by their background under carrier gas only, that is, the molecular interferences were corrected after subtracting the zeros. In jumping acquisition sequences, the $\mathrm{Ca} / \mathrm{Ti}$ and $\mathrm{Cr} / \mathrm{Ti}$ would be best kept as constant as possible to avoid inadequate elemental interference correction at ${ }^{46} \mathrm{Ti},{ }^{48} \mathrm{Ti}$, and ${ }^{50} \mathrm{Ti}$. For example, typical $\mathrm{Cr} / \mathrm{Ti}$ of meteoritic fassaite is about $10^{-3}-10^{-4}$, and an ablation area of a jumping cycle with $10^{-4}$ variation of $\mathrm{Cr} / \mathrm{Ti}$ will yield $6 \varepsilon$ over/under-correcting effect. The best solution to overcome this is to both integrate the Ti signal and monitor the interference simultaneously. To do so requires a mass spectrometer that can cover the mass range of at least from 47 to $53 \mathrm{amu}$. Unfortunately, our mass spectrometer cannot cover such a wide mass range without unacceptable aberration effects.

We also monitored the doubly charged interferences of ${ }^{91} \mathrm{Zr}^{++},{ }^{95} \mathrm{Mo}^{++},{ }^{97} \mathrm{Mo}^{++},{ }^{99} \mathrm{Ru}^{++}$, and ${ }^{105} \mathrm{Pd}^{++}$during analysis at half masses of 45.5, 47.5, 48.5, 49.5, and $52.5 \mathrm{amu}$. We didn't explore the mass discrimination behavior of the doubly charged ion, and there were no doubly charged ions correction developed in our program. The other doubly charged interferences of $\mathrm{Sr}^{++}$and $\mathrm{Cd}^{++}$were also not present. So our acquisition sequences cannot subtract the contributions of ${ }^{88} \mathrm{Sr}^{++}$and ${ }^{106} \mathrm{Cd}^{++}$on the ${ }^{44} \mathrm{Ca}^{+}$and ${ }^{53} \mathrm{Cr}^{+}$signals. According to our experience, signals of doubly charge are less than $5 \%$ relative to their single-charged ion beams. These doubly charged contributions are only less than $0.1 \%$ if these minor or trace elements are only $5 \%$ of titanium concentration in meteoritic titanium rich minerals, such as Ti-pyroxene, hibonite, and perovskite (Meeker et al. 1995). All possible effective interference species are listed in Table 1.

Some other possible interferences observed in the SIMS were also examined. The $\mathrm{SiO}^{+}$species were not found in our background testing while a terrestrial titanium rich biotite mineral was ablated on our system and there was no significant enhancement at 44 amu background. There were also no significant $\mathrm{TiO}^{+}$species found during titanium measurements.

Our instrument optimization procedures consist of using titanium normal standard solution to adjust all mass spectrometer parameters and to optimize signal intensities. Using this approach, we reproduced mass discrimination effects from previous experiment with consistent isotopic 
Table 1. Possible effected molecular and elemental interferences related to Ti isotopic analysis by ICP-MS.

\begin{tabular}{|c|c|c|c|c|}
\hline${ }^{46} \mathrm{Ti}$ & ${ }^{47} \mathrm{Ti}$ & ${ }^{48} \mathrm{Ti}$ & ${ }^{49} \mathrm{Ti}$ & ${ }^{50} \mathrm{Ti}$ \\
\hline \multicolumn{5}{|c|}{ molecular } \\
\hline & & ${ }^{36} \mathrm{Ar}^{12} \mathrm{C}^{+}$ & & ${ }^{38} \mathrm{Ar}^{12} \mathrm{C}^{+}$ \\
\hline & & & & ${ }^{36} \mathrm{Ar}^{14} \mathrm{~N}^{+}$ \\
\hline \multicolumn{5}{|c|}{ elemental } \\
\hline \multirow[t]{3}{*}{${ }^{46} \mathrm{Ca}^{+}$} & & ${ }^{48} \mathrm{Ca}^{+}$ & & \\
\hline & & & & ${ }^{50} \mathrm{~V}^{+}$ \\
\hline & & & & ${ }^{50} \mathrm{Cr}^{+}$ \\
\hline${ }^{92} \mathrm{Zr}^{++}$ & ${ }^{94} \mathrm{Zr}^{++}$ & ${ }^{96} \mathrm{Zr}^{++}$ & & \\
\hline \multirow[t]{2}{*}{${ }^{92} \mathrm{Mo}^{++}$} & ${ }^{94} \mathrm{Mo}^{++}$ & ${ }^{96} \mathrm{Mo}^{++}$ & ${ }^{98} \mathrm{Mo}^{++}$ & ${ }^{100} \mathrm{Mo}^{++}$ \\
\hline & & ${ }^{96} \mathrm{Ru}^{++}$ & ${ }^{98} \mathrm{Ru}^{++}$ & ${ }^{100} \mathrm{Ru}^{++}$ \\
\hline
\end{tabular}

ratios. The laser ablation system was then connected with MC-ICP-MS and used our terrestrial sphene as standard to maximum the signal by tuning the $\mathrm{Ar}$ carrier gas flow only and maintain this setting for all rest of LA-MC-ICP-MS analysis. In practice, each sphene run required seven sets of raw data to obtain its $\mathrm{Ti}$ isotopic ratios and the meteoritic samples would leave them ablated until either the laser raster pattern was completed or Ti signal decreased to levels below detection.

Chromium stable isotope measurements were acquired by the static time-resolved mode that could subtract the background spectrum of solvent directly. The instrumental mass discrimination coefficient was determined by normalizing to the reference ratio of ${ }^{50} \mathrm{Cr} /{ }^{53} \mathrm{Cr}$ from Shields et al. (1966) and used to derive the rest of chromium isotopic ratios. This mass discrimination coefficient was used to compare the coefficient derived from different titanium reference ratios. However, the significant molecular interferences of ${ }^{40} \mathrm{Ar}^{12} \mathrm{C}$ and ${ }^{40} \mathrm{Ar}^{14} \mathrm{~N}$ are about $4 \%$ of ${ }^{52} \mathrm{Cr}$ and $30 \%$ of ${ }^{54} \mathrm{Cr}$ signals respectively, making it difficult to determine their isotopic compositions precisely.

\section{RESULTS}

\subsection{Ti Isotopic Composition of Standards and Analytical Precision}

The titanium isotopic ratios of the $25 \mathrm{ppb}$ Ti standard solution and the four mixed solutions and five terrestrial solids were measured in our MC-ICP-MS with mass discrimination correction by normalizing ${ }^{47} \mathrm{Ti} /{ }^{49} \mathrm{Ti}$. The results are given as fractional deviations in units of epsilon relative to the normal ratios of NPW in Table 2 and Fig. 1. Twice the standard deviations $(2 \times \mathrm{SD}, 2 \sigma)$ of the population were given after the " \pm " sign in Table 2 and plotted as the error bars in Fig. 1. Immediately following the $2 \sigma$ in Table 2, we also showed two times the standard errors (i.e., $2 \times \mathrm{SE}, 2 \sigma_{e}$ ), defined as $[(\mathrm{SD}) /($ square-root of number of repeats)] also termed as "the SD of the mean." For instance, the most frequently repeated sample for our experiment is the standard solution. For the total of 207 repeat analyses, the mean ${ }^{46} \mathrm{Ti} /{ }^{49} \mathrm{Ti}$ is $-1.4 \varepsilon$ with a $2 \sigma$ of $2.4 \varepsilon$ thus we expect the next repeat should fall between -3.8 and $1.0 \varepsilon$. The $2 \sigma_{e}$ is 0.2 and the true ${ }^{46} \mathrm{Ti} /{ }^{49} \mathrm{Ti}$ estimated from the repeats would fall between -1.6 to $-1.2 \varepsilon$. Therefore the difference between its mean of $-1.4 \varepsilon$ and the TIMS ratio used as the reference zero is too large to be entirely due to uncertainty since NPW gave a $95 \%$ level of confidence of $0.5 \varepsilon$. Therefore the minimum gap to bridge is $-1.2+0.5=0.7 \varepsilon$ making it extremely unlikely that the two measurements gave the same answer. Even more conflicting results were obtained for ${ }^{48} \mathrm{Ti} /{ }^{49} \mathrm{Ti}$. The highest value for this ratio would be $-1.7+0.1=-1.6 \varepsilon$ which is still far away from the zero whose uncertainty is only $0.5 \varepsilon$, leaving a gap of $1.1 \varepsilon$. The origin of this $-1.7 \varepsilon$ offset is unclear. We speculate that it may be related to the different methods to take the zero and background; but how to address the issues requires more investigation.

The mean ${ }^{50} \mathrm{Ti} /{ }^{49} \mathrm{Ti}$ has a large offset of $13.5 \varepsilon$ with a $2 \sigma$ of $3.1 \varepsilon$ and $2 \sigma_{e}$ of $0.2 \varepsilon$. We suspect that it was due to the use of Nier's oxygen isotope ratios $\left({ }^{18} \mathrm{O} /{ }^{16} \mathrm{O}=0.002045\right)$ in their correction for oxide interference. If they have used the oxygen composition they measured in that instrument $\left({ }^{18} \mathrm{O} /{ }^{16} \mathrm{O}=\right.$ $0.001997)$ instead the offset would decrease to $5.2 \varepsilon$. According to the above, we suggested the TIMS data should use its own instrumental ${ }^{18} \mathrm{O} /{ }^{16} \mathrm{O}$ ratio when correcting for oxide interference. Since we referenced all ablation data to our sphene working standard, this systematic offset can be cancelled. The deviation or discrepancy of titanium isotopic ratios among several groups will not influence the results for natural samples.

The titanium ratios of four mixed solutions showed that our procedures can effectively correct for calcium and chromium interferences on titanium isotopic measurements. 
Table 2. Ti isotopic ratios of standard solution with different element mixtures and several terrestrial mineral/synthetic standards.

\begin{tabular}{|c|c|c|c|}
\hline Mass Discrimination $\left(\% \mathrm{amu}^{-1}\right)$ & $\varepsilon^{46} \mathbf{T i} /{ }^{49} \mathbf{T i}$ & $\varepsilon^{48} \mathbf{T i} /{ }^{49} \mathrm{Ti}$ & $\varepsilon^{50} \mathrm{Ti} /{ }^{49} \mathrm{Ti}$ \\
\hline \multicolumn{4}{|l|}{ Solution reagent: } \\
\hline \multicolumn{4}{|c|}{ Ti Standard Solution $-\mathrm{Ti} \sim 25 \mathrm{ppb}[207]^{\mathrm{a}}$} \\
\hline $3.314 \pm 0.062^{\mathrm{b}}$ & $-1.4 \pm 2.4(0.2)^{\mathrm{c}}$ & $-1.7 \pm 1.7(0.1)$ & $13.5 \pm 3.1(0.2)$ \\
\hline \multicolumn{4}{|c|}{ Ti-Cr Mixture $(\mathrm{Ti} / \mathrm{Cr} \sim 1300)-\mathrm{Ti} \sim 50 \mathrm{ppb}[1]$} \\
\hline $3.300 \pm 0.002$ & $1.3 \pm 0.6(0.6)$ & $0.8 \pm 0.5(0.5)$ & $11.3 \pm 1.0(1.0)$ \\
\hline \multicolumn{4}{|c|}{ Ti-Cr Mixture $(\mathrm{Ti} / \mathrm{Cr} \sim 130)-\mathrm{Ti} \sim 70 \mathrm{ppb}[8]$} \\
\hline $3.240 \pm 0.020$ & $0.9 \pm 1.0(0.4)$ & $0.9 \pm 0.4(0.1)$ & $10.2 \pm 1.4(0.5)$ \\
\hline \multicolumn{4}{|c|}{ Ca-Ti Mixture $(\mathrm{Ca} / \mathrm{Ti} \sim 5)-\mathrm{Ti} \sim 35 \mathrm{ppb}[8]$} \\
\hline $3.207 \pm 0.020$ & $-1.7 \pm 2.8(1.0)$ & $1.0 \pm 0.7(0.2)$ & $11.2 \pm 2.6(0.9)$ \\
\hline \multicolumn{4}{|c|}{ Simulated Fassaite Solution (Ca:Ti:Al:Mg:Cr 6:2:3:2:0.0007) — Ti $30 \mathrm{ppb}[3]$} \\
\hline $3.242 \pm 0.030$ & $-1.1 \pm 2.2(1.3)$ & $1.1 \pm 0.6(0.3)$ & $11.7 \pm 2.1(1.2)$ \\
\hline
\end{tabular}

Solid standards:

Titanium Metal (Ti) [32]
$3.212 \pm 0.087$
$-0.1 \pm 1.7(0.3)$
$0.9 \pm 0.9(0.2)$
$11.6 \pm 2.5(0.4)$

Rutile (TiO2) [52]
$3.193 \pm 0.06$
$0.8 \pm 2.9(0.4)$
$0.5 \pm 1.1(0.2)$
$11.9 \pm 2.2(0.3)$

Perovskite (CaTiO3) [9]
$3.142 \pm 0.034$
$-1.4 \pm 2.5(0.8)$
$0.5 \pm 1.0(0.3)$
$14.5 \pm 4.6(1.5)$

Sphene (CaTiSiO5) [68]
$3.160 \pm 0.064$
$0.5 \pm 2.2(0.3)$
$0.8 \pm 1.4(0.2)$
$12.6 \pm 2.9(0.4)$

Man-Made Fassaite Glass [5]

$$
3.082 \pm 0.032
$$

$-2.7 \pm 2.3(1.0)$

$-0.3 \pm 1.1(0.5)$

$10.5 \pm 2.5(1.1)$

a: The brackets with number behind analyzed solutions/standards are the measured numbers for statistics in the experiments.

$b$ : Those numbers are $2 \times$ of standard deviation of the population $(2 \sigma)$ of each reagent/standard.

c: The numbers inside the bracket are $2 \times$ of standard deviation of the mean $\left(2 \sigma_{e}\right)$ of each reagent/standard. 
(a)

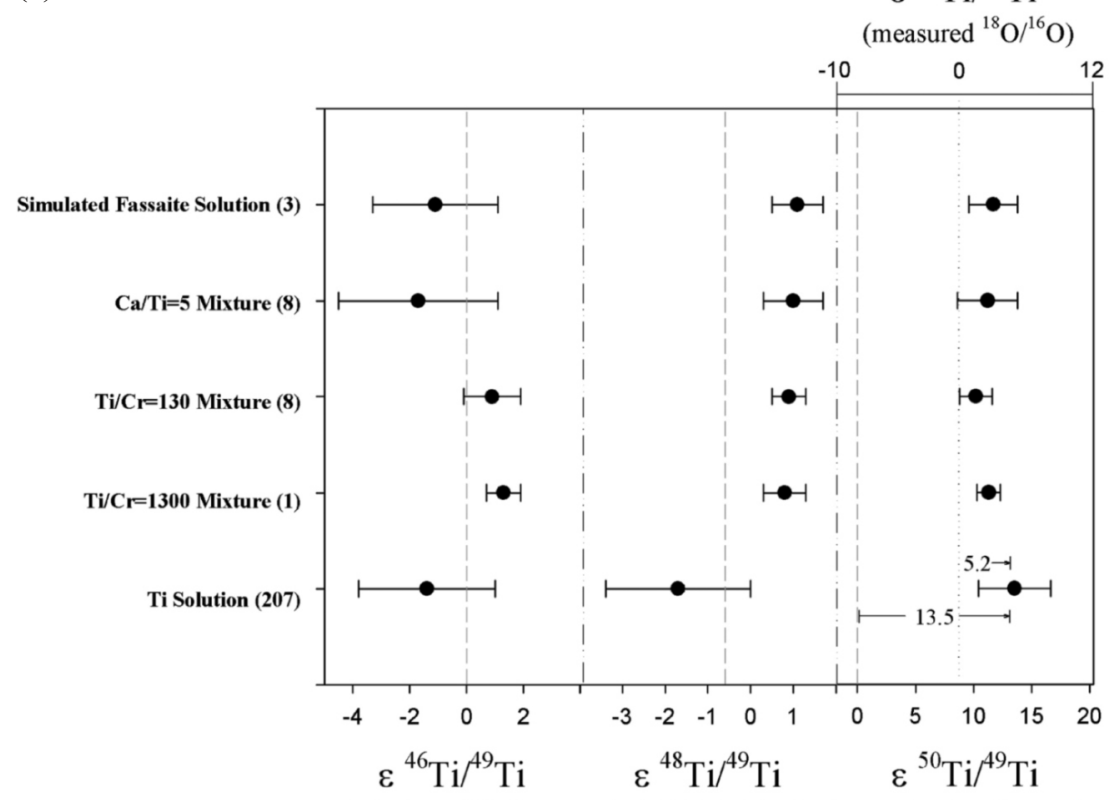

(b)

Relative to Niederer et al. (1981) by Nier's Oxygen Ratios Correction

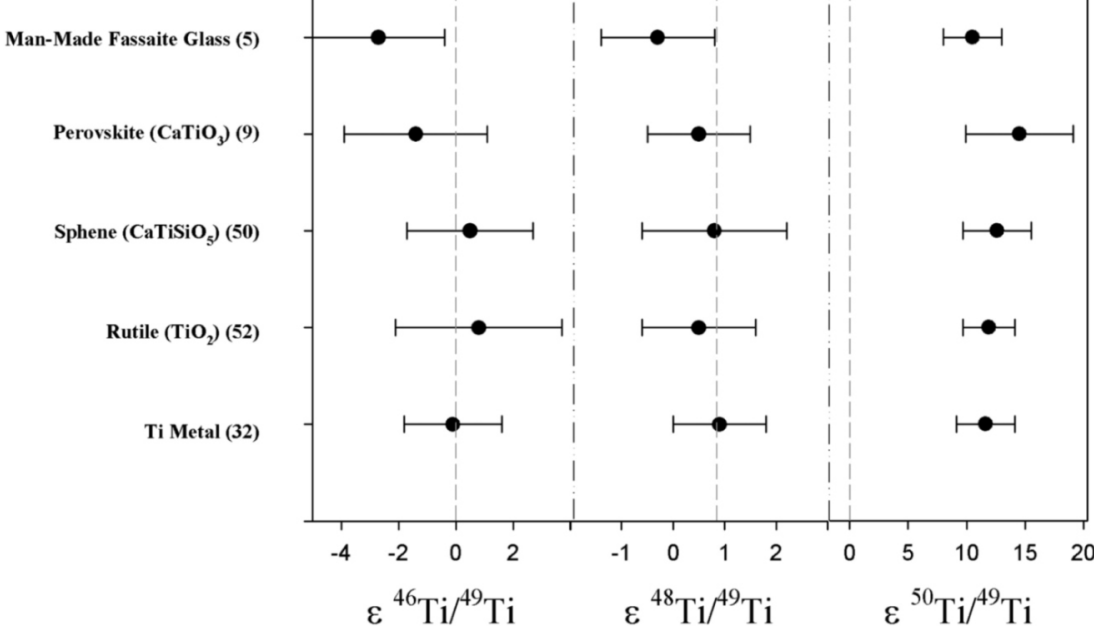

Fig. 1. Fractionation corrected Ti isotopic composition for 5 standard solutions (a) and 5 terrestrial solids (b). The number in the parenthesis after each sample is the total number of repeats for that sample. The grand mean of the fractional deviation in epsilon units relative to the normal ratios of NPW (1981) are plotted for each sample. The error bars are 2 standard deviations of the population. For ${ }^{46} \mathrm{Ti} /{ }^{49} \mathrm{Ti}$ and ${ }^{48} \mathrm{Ti} /{ }^{49} \mathrm{Ti}$ both solutions and the ablated solids gave ratios consistent with the normal ratios. But for ${ }^{50} \mathrm{Ti} /{ }^{49} \mathrm{Ti}$ all samples showed a $13.5 \varepsilon$ shifts. This shift would be reduced to $5.2 \pm 3.1 \varepsilon$ if the oxygen isotopic composition measured by NPW in their mass spectrometer is used to correct for the oxide interference instead of the Nier's value. We used the standard bracket sample method, and the $2 \times \mathrm{SD}$ of the sphene working standards was considered to be the minimum error.

Table 2 presents results for the five solid phase samples Ti metal, rutile $\left(\mathrm{TiO}_{2}\right)$, perovskite $\left(\mathrm{CaTiO}_{3}\right)$, sphene $(\mathrm{CaTiSiO})$, and fassaite glass (to mimick matrix effects). We note that the five solids all gave results close to the normal values during ablation. This result confirms that the isotopic ratios were independent from target species among different titanium bearing minerals during mass spectrometry and the correction procedures for both elemental and molecular interference were effective.
Figure 2 is a histogram of $\varepsilon^{50} \mathrm{Ti} /{ }^{49} \mathrm{Ti}$ for our 68 repeats of sphene it clearly shows the Gaussian distribution of ${ }^{50} \mathrm{Ti} /{ }^{49} \mathrm{Ti}$ ratios after mass discrimination correction. Although the standard deviation of the population should stay the same as number of repeats increase, the uncertainty of the grand mean, as represented by the two standard errors, would decrease as $1 /$ (square root of the total number of repeats). The $2 \sigma$ is $2.9 \varepsilon$. The $2 \sigma_{e}$ near the top is only about $0.4 \varepsilon$. Figure 3 plots the 68 runs of Sphene in the chrono- 


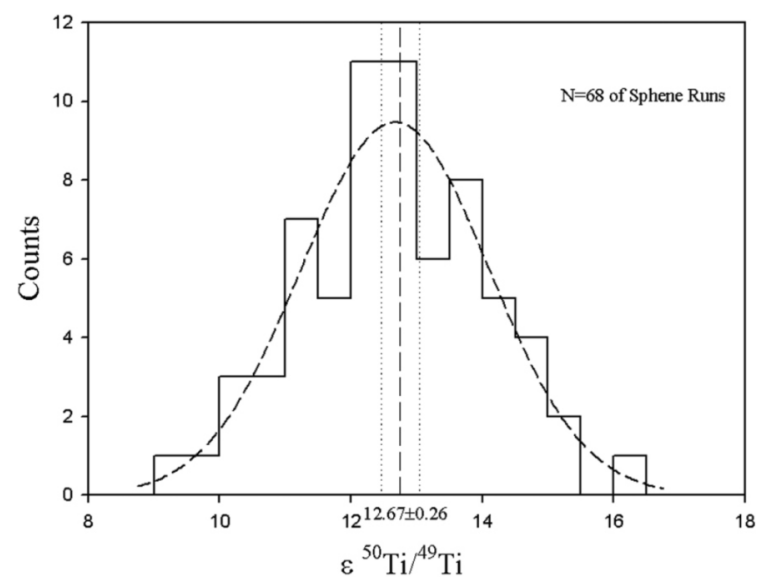

Fig. 2. Histogram of $\varepsilon^{50} \mathrm{Ti} /{ }^{49} \mathrm{Ti}$ for the 68 repeats of the sphene working standard. This distribution appears to be Gaussian with a grand mean of about $12.67 \varepsilon$ and 2 standard deviations of the population around $2.85 \varepsilon$. The 2 standard errors $(0.26 \varepsilon)$ are also plotted.

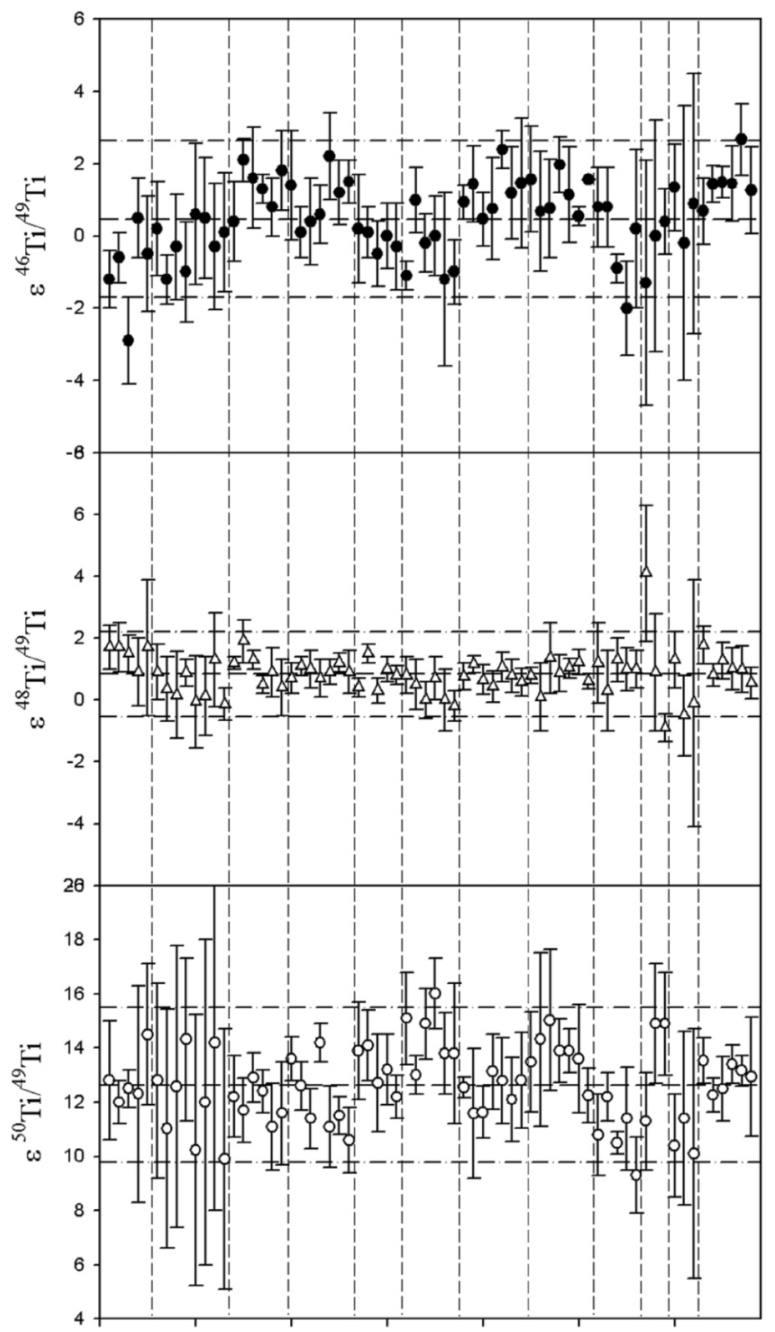

Fig. 3. Titanium isotopic ratios of terrestrial sphene standard were monitored continuously. Most of the time the stability of MC-ICP-MS was acceptable but occasionally sudden changes occurred without warning (See the data point near the left of $\varepsilon^{46} \mathrm{Ti} /{ }^{49} \mathrm{Ti}$ plot and right of $\left.\varepsilon^{48} \mathrm{Ti} /{ }^{49} \mathrm{Ti}\right)$. logical order. The vertical broken lines between two consecutive-day measurements, show some minor but discernable changes from day to day. In order to minimize their effect on the extraterrestrial samples, we have decided to run standards and samples sequentially, depicted in Fig. 4, as the routine instrumental procedure for real samples. Sphene was used as our working standard. We first tuned the MS using a solution standard. We adjusted the ablation using sphene to verify stability until we recovered our normal operating conditions. We then went into the bracket mode by alternating sample and standard. At the end of each day we

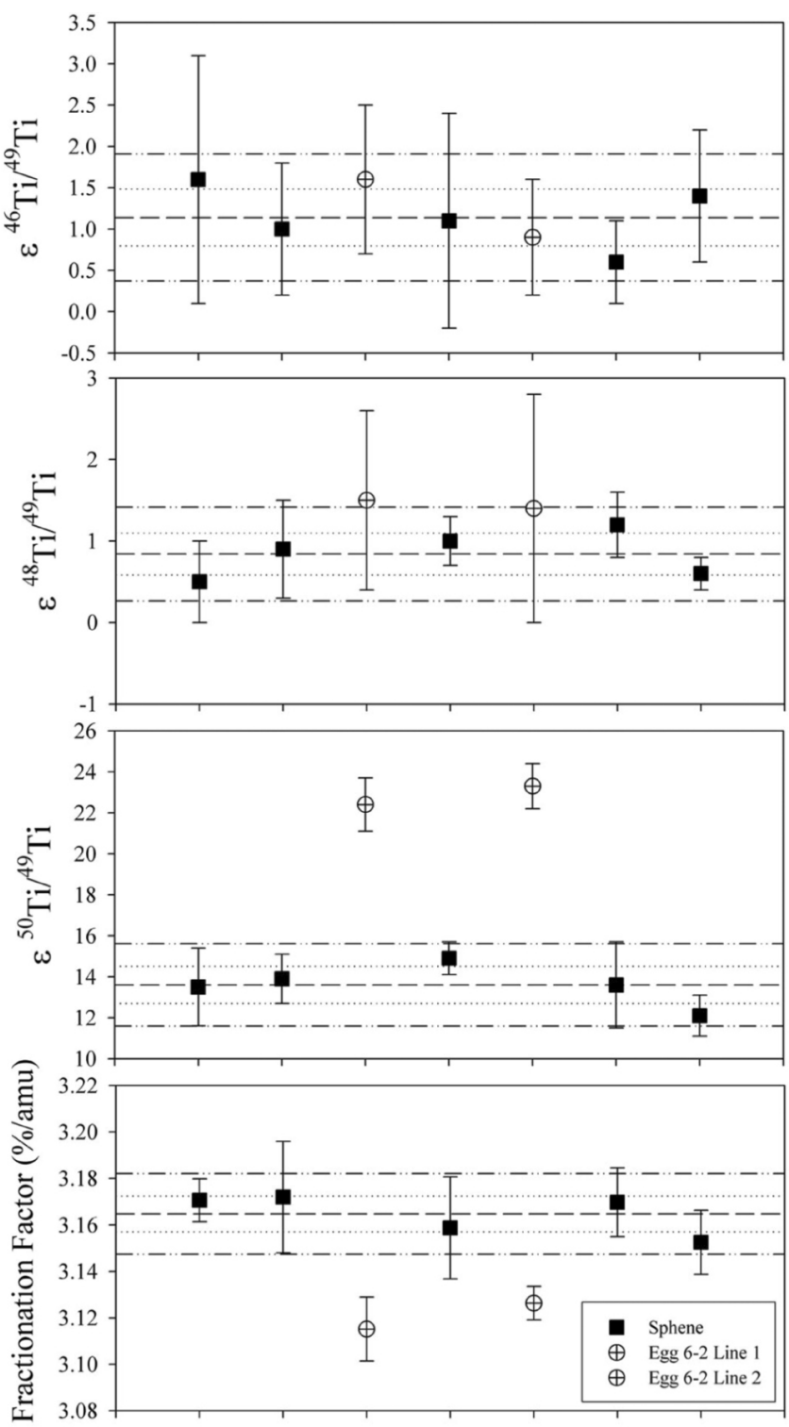

Fig. 4. The current data based on bracketing the samples with standards. Two lines in one fassaite grain from the Egg6 of Allende CAI were measured interspersed between five standards whose grand means will be used as the normal value and the uncertainties will be given as 2 standard deviations of the mean of either all bracketing sphene standard runs or individual measurement of fassaite grain, whichever is larger. Therefore, the uncertainty can be entirely from the 2SD of the sphene standard population. The values for 68 repeats were $2.2 \varepsilon$ for ${ }^{46} \mathrm{Ti} /{ }^{49} \mathrm{Ti}, 1.4 \varepsilon$ for ${ }^{48} \mathrm{Ti} /{ }^{49} \mathrm{Ti}$ and $2.9 \varepsilon$ for ${ }^{50} \mathrm{Ti} /{ }^{49} \mathrm{Ti}$ respectively. 
repeated 2 - 3 standard sphenes to show that the LA-MCICP-MS was under control. The mean of all sphene ratios analyzed was used to distinguish isotopic anomalies in samples. We compared the $2 \sigma$ of the sphene population run that day with the internal $2 \sigma_{e}$ of the sample run and used the larger as the uncertainty for that sample.

\subsection{Ti Isotopic Ratios of a Meteoritic Fassaite}

Two bands on a fassaite grain (\#2) from the Allende CAI Egg-6 were ablated to determine their Ti isotopic ratios. Both analyses gave similar Ti signals as the standard, and the precision was similar to that for standards. In Fig. 4 and Table 3, we show that the 5 sphene runs gave a mean ${ }^{50} \mathrm{Ti} /{ }^{49} \mathrm{Ti}$ of $13.6 \varepsilon$ and $2 \sigma$ of $2.0 \varepsilon$, and the final answer for the two bands on Egg-6 (\#2) were 8.8 and $9.7 \varepsilon$ whose weighted mean of $9.3 \varepsilon$ agrees well with the TIMS result. The weighted ${ }^{46} \mathrm{Ti} /{ }^{49} \mathrm{Ti}$ and ${ }^{48} \mathrm{Ti} /{ }^{49} \mathrm{Ti}$ of Egg-6 (\#2) fassaite were $0.1 \pm 0.8$ and $0.7 \pm 0.9 \varepsilon$, respectively, and also showed consistent results with TIMS work and indicated isotopic normal composition.

\section{DISCUSSION AND CONCLUSION}

\subsection{Matrix Effects}

The constant instrumental mass discrimination behavior of ICP-MS makes it possible to identify natural mass dependent fractionation effects, but the chemical composition of samples will slightly modify isotopic behavior (also called matrix effects) during ion discrimination in the sampler and skimmer cones. The instrumental mass discrimination effect for Ti isotopes seemed to be slightly reduced if the element of interest is mixed with other elements. The range of instrumental fractionation was $3.20-3.31 \%$ per amu using pure titanium and mixed solution standards. The Ti fractionation for the five ablated solid phase standards sys- tem was found to range from $3.08-3.21 \% \mathrm{amu}^{-1}$ which is systematically less than the range for solution standards. Nevertheless, this range of $0.13 \% \mathrm{amu}^{-1}$ for solid standards is comparable to that for solution standards $\left(0.11 \% \mathrm{amu}^{-1}\right)$. This variation is considered as the typical range for fractionation between pure $\mathrm{Ti}$ and fassaite. In Table 3, the natural fractionation factor of meteoritic fassaite was determined by the mass discrimination differences between the meteoritic fassaite and the mean of bracket sphene standards plus the average mass discrimination offset between our sphene and man-made fassaite glass $\left(7.8 \varepsilon \mathrm{amu}^{-1}\right.$, Table 2). Our data $(2.8 \pm 1.7$ and $4.0 \pm 1.7 \varepsilon)$ disagreed with the previous TIMS bulk sample value of $7.4 \pm 1.7 \varepsilon$ (Niederer et al. 1985). This may be due to either some unidentified matrix effect disturbing mass discrimination behavior or variation of natural fractionation effect of the Egg-6 CAI.

\subsection{Inter-Laboratory Inconsistency of Ti Isotopic Ratios}

In order to compare the TIMS results (HFC, NPW, NL, $\mathrm{N}$, and IUPAC) on the normal terrestrial standards with our MC-ICP-MS values, we took their data for terrestrial standards and renormalized them to the ${ }^{47} \mathrm{Ti} /{ }^{49} \mathrm{Ti}$ ratio to obtain the fractionation factors and corrected isotopic ratios for ${ }^{46} \mathrm{Ti},{ }^{48} \mathrm{Ti}$, and ${ }^{50} \mathrm{Ti}$ relative to ${ }^{49} \mathrm{Ti}$ by our 207 times measurements of $25 \mathrm{ppb}$ Ti standard solution. The mass discrimination effect derived from the NPW reference is closest with that for chromium (Table 4), and the normalized titanium isotopic ratios are also quite consistent with the TIMS measurements excepting that discrepancy of $13.5 \pm 3.1 \varepsilon$ higher than NPW at ${ }^{50} \mathrm{Ti} /{ }^{49} \mathrm{Ti}$. The HFC gives the most consistent results at all $\mathrm{Ti}$ isotopic ratios with our ICP-MS measurement, however, the derived instrumental mass discrimination effect was slightly higher than that for chromium. More detailed experiments are required to further explain these results. Mass dis-

Table 3. Ti isotopic ratios of Allende CAI Egg6-2 fassaite grain determined by our LA-MC-ICPMS technique.

\begin{tabular}{|c|c|c|c|c|c|}
\hline Analyzed Sample & $\mathbf{F F}\left(\varepsilon \mathrm{amu}^{-1}\right)^{\mathrm{a}}$ & $\varepsilon^{46} \mathrm{Ti}^{49} \mathrm{Ti}^{\mathrm{b}}$ & $\varepsilon^{48} \mathrm{Ti}^{49} \mathrm{Ti}^{\mathrm{b}}$ & $\varepsilon^{50} \mathrm{Ti}^{49} \mathrm{Ti}^{\mathrm{b}}$ & ${ }^{48} \mathrm{Ti}$ intensity $\left(10^{-11} \mathrm{Amp}\right)$ \\
\hline Egg6-2 Line1 & $2.8 \pm 1.7$ & $0.5 \pm 0.9$ & $0.6 \pm 1.1$ & $8.8 \pm 2.0$ & $3.5 \pm 0.2$ \\
\hline Egg6-2 Line2 & $4.0 \pm 1.7$ & $-0.3 \pm 0.8$ & $0.5 \pm 1.4$ & $9.7 \pm 2.0$ & $3.7 \pm 0.1$ \\
\hline Egg6 (TIMS) & $7.4 \pm 1.7$ & $-1.0 \pm 0.7$ & $1.9 \pm 1.1$ & $9.3 \pm 1.6$ & \\
\hline
\end{tabular}


the solar system. Astrophys. J., 313, 420-428, doi: 10.1086/164980. [Link]

Horn, I. and D. Günther, 2003: The influence of ablation carrier gasses $\mathrm{Ar}, \mathrm{He}$, and $\mathrm{Ne}$ on the particle size distribution and transport efficiencies of laser ablation-induced aerosols: Implications for LA-ICP-MS. Appl. Surf. Sci., 207, 144157, doi: 10.1016/S0169-4332(02)01324-7. [Link]

Hutcheon, I. D., I. M. Steele, D. E. S. Wachel, J. D. MacDougall, and D. Phinney, 1983: Extreme MG fractionation and evidence of TI isotopic variations in Murchison refractory inclusions. Lunar Planet. Sci., 14, 339-340.

Ireland, T. R., W. Compston, and H. R. Heydegger, 1985: Titanium isotopic anomalies in hibonites from the Murchison carbonaceous chondrite. Geochim. Cosmochim. Acta, 49, 1989-1993, doi: 10.1016/0016-7037(85)90092-4. [Link]

Lee, T., 1988: Implications of isotopic anomalies for nucleosynthesis. In: Kerridge, J. F. and M. S. Matthews (Eds.), Meteorites and the Early Solar System, University of Arizona Press, 1063-1089.

Leya, I., M. Schönbächler, U. Wiechert, Urs Krähenbühl, and A. N. Halliday, 2007: High precision titanium isotope measurements on geological samples by high resolution MC-ICPMS. Int. J. Mass Spectrom., 262, 247-255, doi: 10.1016/j.ijms.2006.12.001. [Link]

Mank, A. J. G. and P. R. D. Mason, 1999: A critical assessment of laser ablation ICP-MS as an analytical tool for depth analysis in silica-based glass samples. J. Anal. At. Spectrom, 14, 1143-1153, doi: 10.1039/a903304a. [Link]

Meeker, G. P., 1995: Constraints on formation processes of two coarse-grained calcium-aluminum-rich inclusions: A study of mantles, islands and cores. Meteoritics, 30, 71-84.

Niederer, F. R., D. A. Papanastassiou, and G. J. Wasserburg, 1980: Endemic isotopic anomalies in titanium. Astrophys. J., 240, L73-L77, doi: 10.1086/183326. [Link]

Niederer, F. R., D. A. Papanastassiou, and G. J. Wasserburg, 1981: The isotopic composition of titanium in the Allende and Leoville meteorites. Geochim. Cosmochim. Acta, 45, 1017-1031, doi: 10.1016/0016-7037(81)90128-9. [Link]

Niederer, F. R., D. A. Papanastassiou, and G. J. Wasserburg, 1985: Absolute isotopic abundances of $\mathrm{Ti}$ in meteorites. Geochim. Cosmochim. Acta, 49, 835-851, doi: 10.1016/ 0016-7037(85)90176-0. [Link]

Niemeyer, S., 1988: Titanium isotopic anomalies in chondrules from carbonaceous chondrites. Geochim. Cosmochim.
Acta, 52, 309-318, doi: 10.1016/0016-7037(88)90086-5. [Link]

Niemeyer, S. and G. W. Lugmair, 1981: Ubiquitous isotopic anomalies in Ti from normal Allende inclusions. Earth Planet. Sci. Lett., 53, 211-225, doi: 10.1016/0012-821X (81)90155-2. [Link]

Niemeyer, S. and G. W. Lugmair, 1984: Titanium isotopic anomalies in meteorites. Geochimi. Cosmochim. Acta, $\mathbf{4 8}$ 1401-1416, doi: 10.1016/0016-7037(84)90398-3. [Link]

Nier, A. O., 1950: A redetermination of the relative abundances of the isotopes of carbon, nitrogen, oxygen, argon, and potassium. Phys. Rev., 77, 789-793, doi: 10.1103/PhysRev. 77.789. [Link]

Papanastassiou, D. A. and C. A. Brigham, 1989: The identification of meteorite inclusions with isotope anomalies. Astrophys. J., 338, L37-L40, doi: 10.1086/185395. [Link]

Rosman, K. J. R. and P. D. P. Taylor, 1998: Isotopic compositions of the elements 1997. Pure Appl. Chem., 70, 217235, doi: 10.1351/pac199870010217. [Link]

Russell, W. A., D. A. Papanastassiou, and T. A. Tombrello, 1978: Ca isotope fractionation on the Earth and other solar system materials. Geochim. Cosmochim. Acta, 42, 10751090, doi: 10.1016/0016-7037(78)90105-9. [Link]

Shahar, A. and E. D. Young, 2007: Silicon isotope ratios analysis of a CAI by laser ablation MC-ICPMS and implications for the astrophysics of CAI formation. Lunar Planet. Sci., 38, 1445.

Shields, W. R., T. J. Murphy, E. J. Cantanzaro, and E. L. Garner, 1966: Absolute isotopic abundance ratios and the atomic weight of a reference sample of chromium. J. Res. Nat. Bur. Stand., 70A, 193-197.

Simon, J. I., E. Tonui, S. S. Russell, and E. D. Young, 2004: Mg isotopes zonation in CAIs - new constraints on CAI evolution. Lunar Planet. Sci., 35, 1668.

Young, E. D., R. D. Ash, A. Galy, and N. S. Belshaw, 2002: Mg isotope heterogeneity in the Allende meteorite measured by UV laser ablation-MC-ICPMS and comparisons with $\mathrm{O}$ isotopes. Geochim. Cosmochim. Acta, 66, 683-698, doi: 10.1016/S0016-7037(01)00796-7. [Link]

Zhu, X. K., A. Makishima, Y. Guoa, N. S. Belshaw, and R. K. O’Nions, 2002: High precision measurement of titanium isotope ratios by plasma source mass spectrometry. Int. J. Mass Spectrom., 220, 21-29, doi: 10.1016/S1387-3806 (02)00767-4. [Link] 